\title{
Estimating the fracture density of small-scale vertical fractures when large-scale vertical fractures are present
}

\author{
Yuwei Liu*, Jilin University, Mike Fehler, Xinding Fang, MIT and Cai Liu, Jilin University
}

\section{SUMMARY}

When fractures are vertical, aligned and their dimensions are small relative to the seismic wavelength, the medium can be considered to be an equivalent Horizontal Transverse Isotropic (HTI) medium. However, geophysical data acquired over naturally fractured reservoirs often reveal the presence of multiple fracture sets. We investigate a case where there are two vertical sets of fractures having differing length scales. One fracture set has length scale that is much smaller than the seismic wavelength but the other has length scale that is similar to the seismic wavelength. We use synthetic data to investigate the ability to infer the properties of the small-scale fractures in the presence of the large-scale fracture set. We invert for the Thomsen-type anisotropic coefficients of the small-scale fracture set by using the difference of the P-wave amplitudes at two azimuths, which makes the inversion convex. Then we investigate the influence of the presence of the large-scale fractures on our ability to infer the properties of the small-scale fracture set. Surprisingly, we find that we can reliably infer the fracture density of the small scale fractures even in the presence of large scale fractures having significant compliance values.

\section{INTRODUCTION}

There are several approaches for modeling the seismic response of fractured medium. The effective medium approach is the most commonly used when fracture dimesions and spacings are small relative the seismic wavelength. Hudson theory (Hudson, 1980, 1981, 1988) is a model for isolated oblate spheroidal penny-shaped cracks embedded in an isotropic solid. Linearslip theory (Schoenberg, 1980, 1983) considers that fractures have an excess compliance that leads to an effective compliance tensor of the medium that includes fractures. Then the background and fracture parameters can be related to the Thomsentype anisotropic coefficients (Rüger, 1997; Thomsen, 1986), which describe the influence of anisotropy on various seismic signatures. When fractures are much larger than the seismic wavelength, the fractures will scatter the incident seismic waves and generate complex scattering codas.

Rocks with vertical cracks or fractures can be considered as equivalent HTI media. This provides an opportunity to extract the Thomsen-type anisotropic parameters from seismic waves by measuring Amplitude variation with Azimuth and Offset (AVOZ). However, when fracture sets having differing orientations exist, the seismic amplitude response will be more complicated. In this paper, we assume that the isotropic host rock has a vertical crack set whose dimensions are smaller than the seismic wavelength and a vertical fracture set whose dimensions are larger than the seismic wavelength.

The main objective of this study is to investigate the reli- ability of inverting AVOZ measurements for the anisotropy introduced by the small-scale fractures, and thus characterize the small-scale fractures, when large-scale fractures are also present. We also have interest in characterizing the largescale fractures using a method like Fracture Transfer Function (Fang et al., 2012). Since the velocity difference between the fast and slow shear waves is related to the crack density of the small-scale fractures, we can evaluate crack density based on shear-wave Thomsen-type anisotropy $\operatorname{parameter}(\gamma)$.

\section{THEORY}

Compressional plane wave reflection coefficient for a HTI medium can be approximately described as a function of the polar incident angle $\theta$ and azimuthal phase angle $\varphi$ in the approximate form as (Rüger, 1997)

$$
\begin{aligned}
R \approx & \frac{\Delta Z}{2 \bar{Z}}+\frac{1}{2}\left\{\frac{\Delta \alpha}{\bar{\alpha}}-\left(\frac{2 \bar{\beta}}{\bar{\alpha}}\right)^{2} \frac{\Delta G}{\bar{G}}+\right. \\
& {\left.\left[\Delta \delta^{(V)}+2\left(\frac{2 \bar{\beta}}{\bar{\alpha}}\right)^{2} \Delta \gamma\right] \cos ^{2} \varphi\right\} \sin ^{2} \theta+} \\
& \frac{1}{2}\left(\frac{\Delta \alpha}{\alpha}+\Delta \varepsilon^{(V)} \cos ^{4} \varphi+\Delta \delta^{(V)} \sin ^{2} \varphi \cos ^{2} \varphi\right) \sin ^{2} \theta \tan ^{2} \theta
\end{aligned}
$$

where, $\alpha$ and $\beta$ are vertical P- and S-wave velocities. $G=\rho \beta^{2}$ is the vertical shear modulus while $Z=\rho \alpha^{2}$ is the vertical Pwave impedance. $\rho$ is the rock density. The $\Delta$ denotes contrast across an interface, e.g. $\Delta Z=Z_{2}-Z_{1}$; bar over a symbol means average quantities, e.g. $\bar{Z}=\frac{1}{2}\left(Z_{2}-Z_{1}\right)$. The index 1 corresponds to the upper medium and the index 2 to the lower medium.

$$
\gamma^{(V)}=-\frac{\gamma}{1+2 \gamma}
$$

where $\gamma$ is the generic Thomsen parameter. $\gamma^{(V)}, \delta^{(V)}, \varepsilon^{(V)}$ are the Thomsen-type anisotropic coefficients for HTI medium.

Choosing two azimuths $\varphi$ and $\varphi+\eta$, the difference of their reflection coefficients $\Delta R$ can be expressed as,

$$
\begin{aligned}
\Delta R= & R(\varphi)-R(\varphi+\eta) \\
= & \Delta \gamma\left(\frac{2 \bar{\beta}}{\bar{\alpha}}\right)^{2}\left[\cos ^{2} \varphi-\cos ^{2}(\varphi+\eta)\right] \sin ^{2} \theta \\
& +\frac{1}{2} \delta^{(V)} \sin ^{2} \theta\left\{\left[\cos ^{2} \varphi-\cos ^{2}(\varphi+\eta)\right]+\right. \\
& {\left.\left[\sin ^{2} \varphi \cos ^{2} \varphi-\sin ^{2}(\varphi+\eta) \cos ^{2}(\varphi+\eta)\right] \tan ^{2} \theta\right\} } \\
& +\frac{1}{2} \Delta \varepsilon^{(V)}\left[\cos ^{4} \varphi-\cos ^{4}(\varphi+\eta)\right] \sin ^{2} \theta \tan ^{2} \theta
\end{aligned}
$$

It is more direct to determine the Thomsen-type anisotropy parameters using Equation 3 than Equation 1, so we invert for 


\section{fracture anisotropy}

Table 1: Model parameters

\begin{tabular}{|l|l|l|}
\hline Parameter & Upper layer & Small-scale fractures(Gas-filled) \\
\hline$\gamma$ & 0 & 0.0974 \\
$\delta^{(V)}$ & 0 & -0.1705 \\
$\varepsilon^{(V)}$ & 0 & -0.1635 \\
$\alpha$ & $4398.9 \mathrm{~m} / \mathrm{s}$ & $5236.2 \mathrm{~m} / \mathrm{s}$ \\
$\beta$ & $2447.4 \mathrm{~m} / \mathrm{s}$ & $2970.0 \mathrm{~m} / \mathrm{s}$ \\
$\rho$ & $2.4 \mathrm{~g} / \mathrm{cm}^{3}$ & $2.59 \mathrm{~g} / \mathrm{cm}^{3}$ \\
$\mathrm{e}$ & 0 & 0.08 \\
\hline
\end{tabular}

Thomsen-type anisotropy parameters using the difference of data at two azimuths (Liu et al., 2012).

The inversion for anisotropy parameters from measured AVAZ data is done using a genetic algorithm. The cost function for the inversion is given by

$$
\begin{aligned}
& \text { fitness }=\sum_{i=1}^{I} \sum_{j=1}^{J} \omega_{i j} \sum_{k=1}^{K}\left[\Delta A\left(\varphi_{i}, \eta_{j}, \theta_{k}\right)-\right. \\
& \left.\frac{\Delta A\left(\varphi_{i}, \eta_{j}, \theta_{k_{0}}\right) \Delta R\left(\varphi_{i}, \eta_{j}, \theta_{k}, \Delta \gamma, \Delta \delta^{(V)}, \Delta \varepsilon^{(V)}\right)}{\Delta R\left(\varphi_{i}, \eta_{j}, \theta_{k_{0}}, \Delta \gamma, \Delta \delta^{(V)}, \Delta \varepsilon^{(V)}\right)}\right]^{2} \\
& (i=1,2, \ldots, I ; j=1,2, \ldots J ; k=1,2, \ldots, K)
\end{aligned}
$$

where

$$
\left|A\left(\varphi_{i}, \eta_{j}, \theta_{k_{0}}\right)\right|=\max \left|A\left(\varphi_{i}, \eta_{j}, \theta_{k}\right)\right| \quad(k=1,2, \ldots, K)
$$

A means measured reflection amplitude and $\Delta A$ is amplitude difference between azimuths $\varphi$ and $\varphi+\eta . \omega_{i j}$ is weight coefficient. Then shear-wave splitting parameter $\gamma^{(V)}$ provides a way to quantify crack density $e$ using (Bakulin et al., 2000).

$$
e=-\frac{3(3-2 g) \gamma^{(V)}}{8}
$$

where $g=\frac{V_{S}^{2}}{V_{P}^{2}}, V_{S}$ and $V_{P}$ are the background P- and S-wave velocities.

\section{MODEL AND SYNTHETIC DATA}

Our model consists of two layers. The upper layer is isotropic while the lower one is an HTI medium equivalent to one containing vertical small-scale fractures whose properties are listed in Table 1. The HTI medium properties are determined from the fracture properties using Hudson theory. Figure 1 shows the variation of reflection coefficients with incident angle and azimuth as determined using equation 1 . The azimuthal variation in the reflection coefficients can be used to find the strike of the fractures. As azimuth increases from 0 degree to 90 degrees, the reflection coefficient value decreases at the same incident angle in our example.

We add a set of vertical large-scale fractures to the lower, HTI, medium and described them by using Linear-slip theory. We assume the normal and tangential compliances of

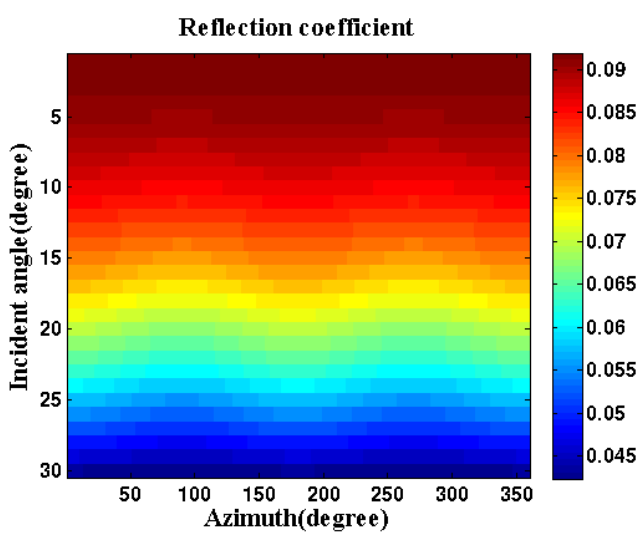

Figure 1: Reflection coefficient vs. incident angle and azimuth for the HTI media(small-scale fractures) used for our study.

the large-scale fractures $\left(Z_{N}\right.$ and $\left.Z_{T}\right)$ are equal. What's more, the strike of large-scale fractures is taken to be either parallel or perpendicular to that of small-scale ones (Fig 2, Fig 3). The large-scale fracture spacing is $50 \mathrm{~m}$. We use a finite difference method (Coates and Schoenberg, 1995; Fang et al., 2013) to generate synthetic data. The scheme allows the simulation of the seismic response in a medium containing large scale fractures embedded within a general HTI medium. We use a Ricker wavelet source with a center frequency of $40 \mathrm{~Hz}$. Thickness of the upper layer is $400 \mathrm{~m}$.

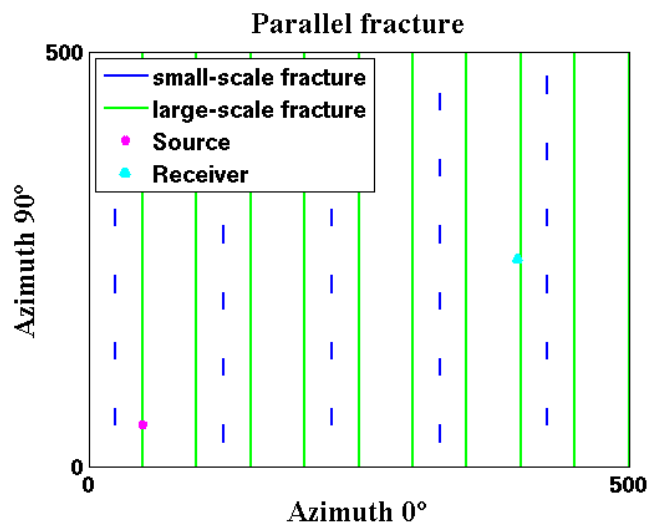

Figure 2: Sketch of parallel fracture model. Offset and azimuth of receiver for traces shown in Figure 4 are $400 \mathrm{~m}$ and 30 degree, respectively.

To study effects of large-scale fractures on our ability to characterize the small-scale fractures, we vary the large-scale fracture compliances from $0.01 \times 10^{-9} \mathrm{~m} / \mathrm{Pa}$ to $0.9 \times 10^{-9} \mathrm{~m} / \mathrm{Pa}$. Figures 4 and 5 show synthetic seismic data for the model where the large-scale fractures strike parallel to the strike of the small scale fractures and where the large-scale fractures are perpendicular to the small-scale fractures, respectively. Reflection amplitude goes down and scattered wave energy goes up as fracture compliance increases. Scattered wave energy is 


\section{fracture anisotropy}

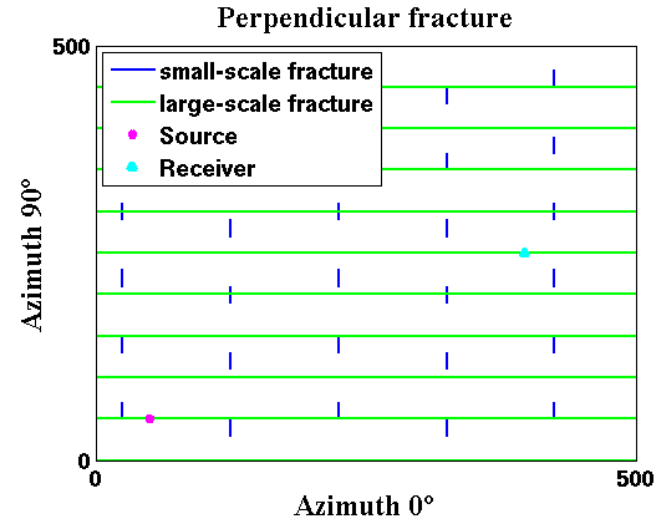

Figure 3: Sketch of perpendicular fracture model.

not remarkable until fracture compliance is larger than $0.1 \times$ $10^{-9}$ for both the parallel fracture model and the perpendicular fracture model. This suggests that large-scale fractures would seriously affect reflection information when large-scale fracture compliance is larger than $0.1 \times 10^{-9} \mathrm{~m} / \mathrm{Pa}$. What's more, the scattered waves caused by perpendicular fractures is much more complicated than that caused by parallel fracture.

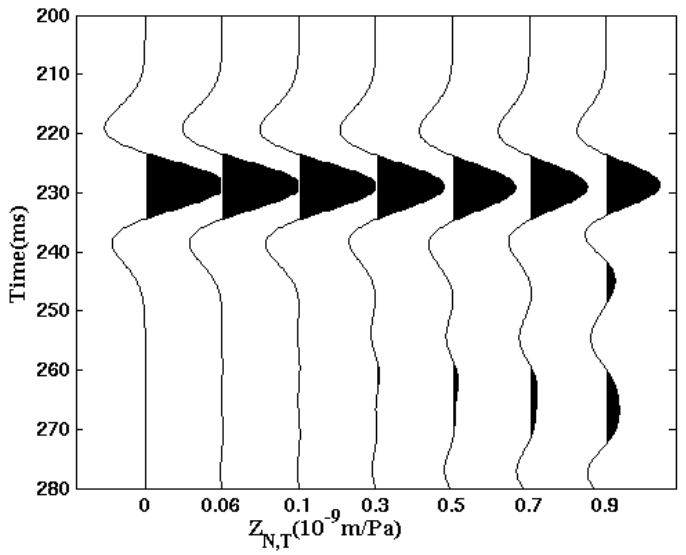

Figure 4: Z-component of motion for the $\mathrm{P}$ wave reflected for the parallel fracture model. Results for compliance varying from 0 (e.g. no large-scale fractures) to $0.9 \times 10^{-9} \mathrm{~m} / \mathrm{Pa}$ are shown. Offset is $400 \mathrm{~m}$.Azimuth is 30 degree.

Figure 6 compares vertical-component waveforms when largescale fracture compliance is $0.6 \times 10^{-9} \mathrm{~m} / \mathrm{Pa}$ at the same receiver. Small-scale fractures dominate the behavior of the first reflection pulse. Absolute amplitude for azimuth 0 degree is larger than for azimuth 90 degree in the first reflection pulse. This result is coincident with what we discussed before when there are only small-scale fractures. However, properties of the scattered waves are mainly governed by large-scale fracture. For the parallel fracture model, amplitude of the scattered wave for azimuth 0 degree is still larger than the one for azimuth 90 degree. For perpendicular fracture model, ampli-

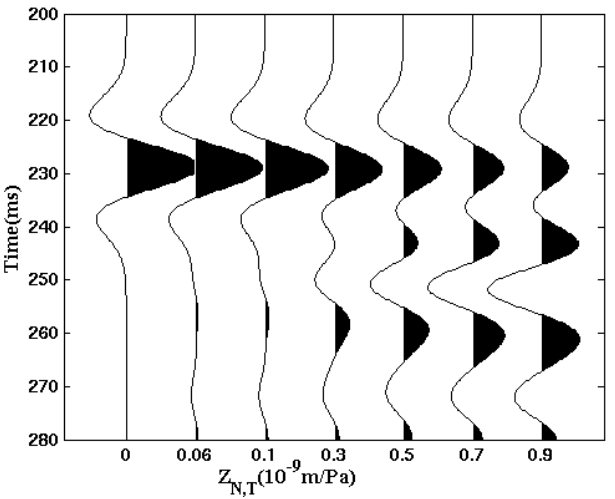

Figure 5: Same as Figure 4 but for perpendicular fracture model.

tude of the scattered wave for azimuth 0 degree is less than the one for azimuth 90 degree. According to our previous discussion, it should be possible to use reflection amplitude azimuth characteristics to evaluate small-scale fracture orientation and the strike of the large-scale fractures can be estimated from the scattered waves.

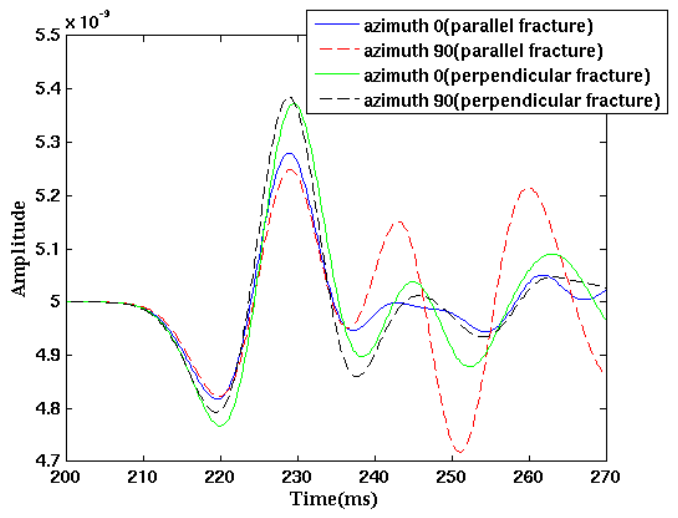

Figure 6: $\mathrm{P}$ wave reflection waveforms( $\mathrm{z}$ component). $Z_{N}, T$ is $0.6 \times 10^{-9} \mathrm{~m} / \mathrm{Pa}$. Offset is $400 \mathrm{~m}$.

\section{INVERSION RESULTS ANALYSIS}

We can, based on Equation 4, invert for Thomsen-type anisotropy parameters $\left(\gamma, \delta^{(V)}\right.$ and $\left.\varepsilon^{(V)}\right)$ by using a genetic algorithm. In Figure 7 and Figure 8, we show the inversion results for parallel fracture and perpendicular fracture models, respectively. It is interesting to note that the inversion results for $\gamma$ are much better than those for the other anisotropy parameters $\left(\delta^{(V)}\right.$ and $\left.\varepsilon^{(V)}\right)$. For the parallel fracture model, the error of the inversion for $\gamma$ is less than $10 \%$ when large-scale fracture compliance is less than $0.1 \times 10^{-9} \mathrm{~m} / \mathrm{Pa}$. Precision of inversion for $\gamma$ decreases when large-scale fracture compliance is more than $0.1 \times 10^{-9} \mathrm{~m} / \mathrm{Pa}$. The maximum error of $\gamma$ is $30 \%$ when large-scale fracture compliance is $0.7 \times 10^{-9} \mathrm{~m} / \mathrm{Pa}$. For the 


\section{fracture anisotropy}

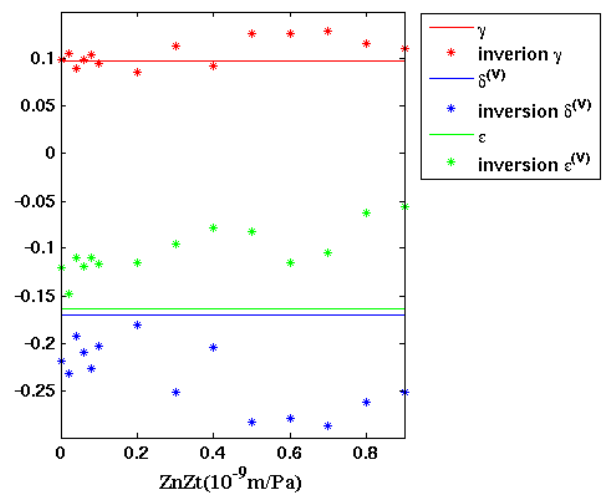

Figure 7: Inversion Thomsen-type anisotropy parameters for parallel fracture model.

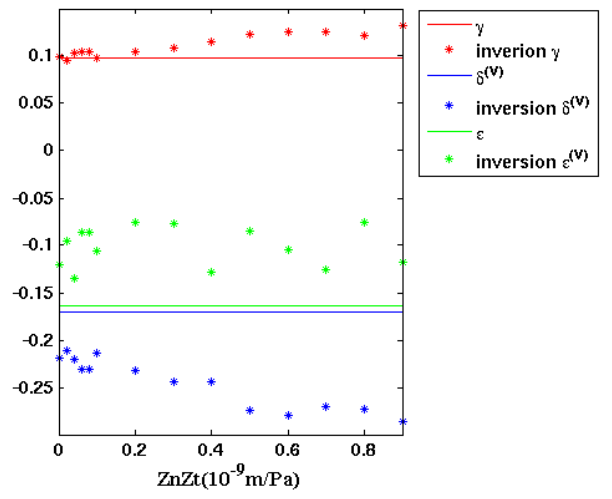

Figure 8: Inversion Thomsen-type anisotropy parameters for perpendicular fracture model.

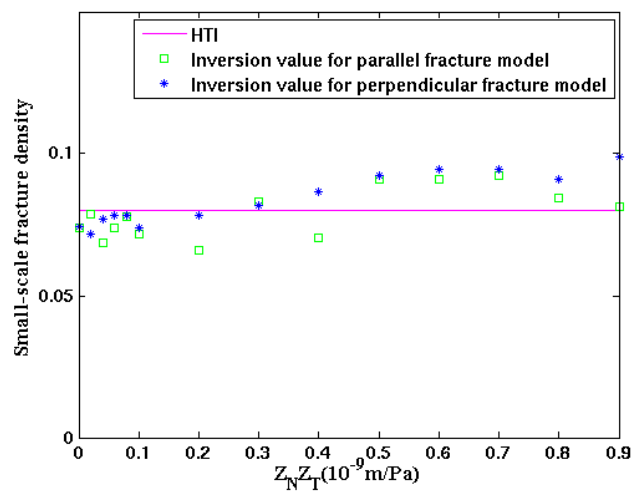

Figure 9: Small-scale fracture density inversion result.

perpendicular fracture model, the error of inversion $\gamma$ is less than $10 \%$ when large-scale fracture compliance is less than $0.2 \times 10^{-9} \mathrm{~m} / \mathrm{Pa}$. The error increases with the growth of fracture compliances larger than $0.2 \times 10^{-9} \mathrm{~m} / \mathrm{Pa}$. The maximum error of $\gamma$ is $35 \%$ when fracture compliance is $0.9 \times 10^{-9} \mathrm{~m} / \mathrm{Pa}$.
According to the relationship between generic Thomsen parameter $\gamma$ and crack density (Equation 2 and Equation 6), we can estimate small-scale fracture density based on inversion for $\gamma$. Figure 9 shows the sensitivity of fracture compliance to estimated crack density. For the parallel fracture model, the error of estimated small-scale fracture density is less than $10 \%$ when large-scale fracture compliance is below $0.1 \times 10^{-9} \mathrm{~m} / \mathrm{Pa}$ expect it is $14 \%$ while large-scale fracture compliance is $0.04 \times$ $10^{-9} \mathrm{~m} / \mathrm{Pa}$. The maximum error of estimated small-scale fracture density is $17 \%$ ocours when fracture compliance is $0.2 \times$ $10^{-9} \mathrm{~m} / \mathrm{Pa}$. For the perpendicular fracture model, the error of estimated small-scale fracture density is less than $10 \%$ when large-scale fracture compliance is less than $0.4 \times 10^{-9} \mathrm{~m} / \mathrm{Pa}$ and the maximum error is $24 \%$ while large-scale fracture compliance is $0.9 \times 10^{-9} \mathrm{~m} / \mathrm{Pa}$. Regardless of parallel fracture and perpendicular fracture, it is almost stable to evaluate smallscale fracture density when large-scale fracture compliance is less than $10^{-10} \mathrm{~m} / \mathrm{Pa}$.

\section{CONCLUSIONS}

We have used numerical simulation and inversion to investigate the ability to infer anisotropy parameters for media containing aligned small-scale and large-scale fractures. The smallscale fractures lead to an equivalent HTI medium and the largescale fractures cause scattering of the seismic waves. We investigated cases where the large-scale fractures are either parallel or perpendicular to the small-scale fractures. Visual inspection of the simulated traces shows that the scattering from the large-scale fractures does not noticeably influence the character of the waveforms when compliance is less than about $10^{-10} \mathrm{~m} / \mathrm{Pa}$. We find that it is possible to use AVOZ inversion to reliably infer the Thomsen anisotropy gamma and hence the fracture density when large-scale fracture compliance is less than about $10^{-10} \mathrm{~m} / \mathrm{Pa}$. While our results depend on several model parameters and the acquisition scenario, they do lead us to believe that AVOZ can often be reliable for estimating the fracture density of small-scale fractures even when large-scale fractures are present.

\section{ACKNOWLEDGMENTS}

Yuwei Liu work was supported by the China Scholarship Council. Additional support came from the Founding Member Program of the Earth Resources Laboratory at MIT. 